». . . und sogar eine alberne Ordnung ist immer noch besser als gar keine.« 
Gerhard Kaiser

\section{». . und sogar eine alberne Ordnung ist immer noch besser als gar keine.«}

Erzählstrategien in Thomas Manns

Doktor Faustus

Verlag J. B. Metzler Stuttgart - Weimar 
Die Deutsche Bibliothek - CIP-Einheitsaufnahme

\section{Kaiser, Gerhard:}

». . . und sogar eine alberne Ordnung ist immer noch besser als gar keine.« :

Erzählstrategien in Thomas Manns Doktor Faustus/Gerhard Kaiser

- Stuttgart ; Weimar : Metzler, 2001

ISBN 978-3-476-45265-8

ISBN 978-3-476-02774-0 (eBook)

DOI 10.1007/978-3-476-02774-0

Dieses Werk einschließlich aller seiner Teile ist urheberrechtlich geschützt. Jede Verwertung außerhalb der engen Grenzen des Urheberrechtsgesetzes ist ohne Zustimmung des Verlages unzulässig und strafbar. Das gilt insbesondere für Vervielfältigungen, Übersetzungen, Mikroverfilmungen und die Einspeicherung und Verarbeitung in elektronischen Systemen.

M \& P Schriftenreihe für Wissenschaft und Forschung

(C) 2001 Springer-Verlag GmbH Deutschland

Ursprünglich erschienen bei J.B.Metzlersche Verlagsbuchhandlung und Carl Ernst Poeschel Verlag GmbH in Stuttgart 2001 


\section{Inhaltsverzeichnis}

Dank

Vorbemerkungen 1

I. Einleitung:

Die Entwicklung der Grundkonzeption des Doktor Faustus

II. „Une mer à boire"

Die Strukturschichten des Romans

und ihr Ermöglichungszusammenhang

1. Schichtungen und Gewichtungen

2. Die Krise der Nation als Krise der Kultur:

Der mentalitätengeschichtliche Ermöglichungszusammenhang und das Deutungsmuster des Autors

2.1 Die Musik als bildungsbürgerliches Paradigma der Kunst

2.2 Die Musik der Moderne und die Problematik

ihrer Stellung im Strukturgefüge des Romans

2.3 Ideengeschichtlicher Ermöglichungszusammenhang:

Analogia entis - Auf der Suche nach der verlorenen

Entsprechung

3. Portrait des Künstlers als moderner Faust?

Die Strukturschicht des Faustromans

4. Der Schleier der Maya:

Die Strukturschicht des Gesellschaftsromans

5. Deutschland. Kein Künstlermärchen:

Die Strukturschicht des Deutschlandromans

6. Zusammenfassung 
1. Grundzüge des Mann'schen Romanverständnisses

2. Zum begrifflichen Instrumentarium der Analyse

3. Analyse der Erzählsituation: Die Autorisierung des Erzählers oder Die Konfirmation des Serenus Zeitblom

3.1 Die Lizenz zum Erzählen 1:

Erzähltyp, Erzählstandort und Sichtweise

3.2 Die Lizenz zum Erzählen 2:

Erzählverhalten und Erzählhaltung

3.3 Der Erzähler und die Stimme seines Autors

4. Strategien der „Musikalisierung“: Die Leitmotivtechnik im Doktor Faustus

4.1 Der Begriff des Leitmotivs bei Thomas Mann

4.2 Der Teufel im Detail - Leitmotive im Doktor Faustus

4.3 „Schwindelige Identitäten“ - Dialektik der Leitmotivtechnik

5. Strategien des "höheren Abschreibens“

Phänomene der Transtextualität im Doktor Faustus

5.1 Ordnung und Kontingenz: Funktion und Grenze mythologisierenden Erzählens im Doktor Faustus

5.2 „Montage“, Anspielung und Pastiche

6. „Doppelte Optik“: Die Welt als Wille zur Vorstellung

IV. „A novel to end all novels"?

Thomas Mann, James Joyce

und die Frage nach der Modernität des Doktor Faustus 
1. „James Joyce“:

Ein semantischer Code zur Rezeptionslenkung 165

2. Exkurs: Doktor Faustus und Ulysses 172

3. Zusammenfassung und Schlußbetrachtung:

Zur Frage nach der Modernität des Doktor Faustus

Anmerkungen

Siglenverzeichnis

235

Literaturverzeichnis

236 


\section{Dank}

Die vorliegende Untersuchung wurde im November 1999 abgeschlossen und vom Fachbereich 3 (Sprach- und Literaturwissenschaften) der Universität-GH Siegen im Wintersemester 1999/2000 als Dissertation angenommen.

Danken möchte ich Prof. Dr. Georg Bollenbeck für die Ermöglichungszusammenhänge und für die ebenso inspirierende wie souveräne Betreuung vor, während und nach meiner Arbeit an dem vorliegenden Buch. Dem Zweitgutachter dieser Arbeit, PD Dr. Jürgen Kühnel, möchte ich sowohl für seine kritischen Anmerkungen und anregenden Hinweise als auch für die Erstellung des gründlichen Gutachtens danken. Desweiteren gilt mein Dank Prof. Dr. Walburga Hülk-Althoff, die freundlicherweise als Dritte die Arbeit mit Interesse begutachtete. Ebenso gedankt sei Marc („mocca grande“) F. Erdl, dessen Geduld, Kenntnisse und freundschaftliche Hilfe maßgeblich bei der Erstellung der Druckfassung waren. Für geduldiges Korrekturlesen und für den Hinweis auf eine mitunter idiosynkratische Interpunktion gilt mein Dank: Edda Bleek und Thomas LaPresti.

Schließlich möchte ich meinen Eltern für die stete Unterstützung danken. 
Wird erst einmal gefragt, ob das Spiel die Mühe lohnt, ist es auch schon aus damit.

Pierre Bourdieu 


\section{Vorbemerkungen}

"Ich anerkenne die moralische Leistung", notiert Thomas Mann am 29.01.1947 zum Abschluß der Niederschrift seines großen Altersromans Doktor Faustus in sein Tagebuch. Die lakonisch anmutende und zugleich von Selbstgefälligkeit nicht freie Einschätzung des Autors mag zwar durchaus zutreffend sein, jedoch erweist sich das so beurteilte Werk - das Ergebnis einer nahezu vierjährigen, von Krisen und Selbstzweifeln des Autors immer wieder gekennzeichneten Schaffensperiode ist - nicht nur als eine außerordentliche moralische, sondern auch als eine ungewöhnliche künstlerische Leistung des zum damaligen Zeitpunkt bereits über siebzigjährigen Autors:

Als moralische Leistung erscheint der Roman nicht zuletzt deshalb, weil er vom Durchhaltewillen und der pedantischen Zähigkeit des Autors zeugt, der um sein körperliches Wohlergehen stets geradezu hypochondrisch besorgt ist und der während der Arbeit am Roman nicht nur von allerlei Alterskränklichkeiten belästigt wird, sondern zudem eine lebensgefährliche LungenkrebsOperation überstehen muß. Als ungewöhnliche künstlerische Leistung zeigt sich der Roman Manns sowohl, weil er, wie G. Lukács bemerkt, "eine monumentale Rekapitulation, Systematisierung aller seiner Jugendthemen"' ist, als auch deshalb, weil der Doktor Faustus seinen Autor erneut auf der schwindelnden Höhe seines stupenden künstlerischen Könnens präsentiert. In virtuoser und geradezu exzessiver Weise spielt Mann in seinem Altersroman noch einmal auf der Klaviatur der ihm zur Verfügung stehenden künstlerischen Mittel, so daß der Doktor Faustus ein reichhaltiges Tableau Mann'scher Erzählstrategien darbietet. Zudem hat der Roman seit seinem Erscheinen vor nunmehr über 50 Jahren (am 17.10.1947 bei Behrmann-Fischer in Stockholm) wie kaum ein zweites Werk innerhalb des umfangreichen Euvres Manns eine Flut an wissenschaftlicher und nicht-wissenschaftlicher Literatur provoziert, die sich mit dem Werk kontrovers auseinandersetzt ${ }^{2}$. Der Doktor Faustus, der das individuelle Schicksal des fiktiven, modernen Komponisten Adrian Leverkühn 
erzählt und zugleich, so Mann, "in der Tiefe beständig vom deutschen Lose"3 handelt, zeitigte und zeitigt ein Rezeptionsverhalten, dessen (Meinungs-) Spektrum von jenen Stimmen, die den Roman als ein "Meisterwerk" einstufen und an ihm "ein fast beängstigendes Angebot an künstlerischem Können, eine bis zum äußersten gesteigerte Vollkommenheit" (E. Fischer) wahrnehmen zu können glauben, bis zu jenen reicht, die das Werk als "geschichtsklitternde Spekulation"4 (H.E. Holthusen), als inhaltlich und formal mißlungenen Versuch Manns bewerten, die Geschichte "Deutschlands und der Deutschen" bis zum katastrophalen Niedergang im 2. Weltkrieg in Romangestalt darzustellen.

Thomas Mann verfolgt neben der feuilletonistischen auch die literaturwissenschaftliche Rezeption seines Spätwerks äußerst angespannt und versucht die Wertungen seines Romans immer wieder zu beeinflussen. Auf Kritiken reagiert er extrem empfindlich und zeigt sich gekränkt. ${ }^{5}$ Als "feindlichen Akt" bezeichnet Mann etwa in einem äußerst maliziösen Brief vom 2.02.48 an Käte Hamburger die Kritik der sonst eher devoten und aufgrunddessen vom Autor geschätzten Literaturwissenschaftlerin, die den Faustus in Manns Augen nicht hinreichend zu schätzen weiß. Mann schließt den Brief mit der von offensichtlicher Verstimmtheit zeugenden Wendung: "Nun kann ich nur hoffen, daß die blühende Zeit ihrem kritischen Talent noch viele ihm besser liegende Werke anbieten möge."6

Noch zwei Jahre vor seinem Tod schreibt der Autor an den Herausgeber eines Almanachs, dessen Frage beantwortend, welches seiner eigenen Bücher ihm am liebsten sei:

Der Faustus-Roman ist mir am teuersten, einfach weil er mich am teuersten zu stehen gekommen ist, mich am meisten Herzblut gekostet hat, weil ich an dies Werk meiner 70 Jahre am meisten von meinem Leben, meinem tiefen Selbst mit einer Art von wilder Rücksichtslosigkeit, einer Aufgewühltheit, die ich nie vergessen werde, dahingegeben habe. Früheres und Späteres von mir, der 'Joseph' etwa oder 'Der Erwählte', mag glücklicher, heiterer, selbst künstlerisch gewinnender sein, - an diesem Buch hänge ich wie an keinem anderen. Wer es nicht mag, den mag ich sogleich nicht mehr. ${ }^{7}$ 
Obwohl der vom Autor angedrohte Sympathieentzug einer heutigen Untersuchung des Romans nicht mehr, möglicherweise die Klarsicht hemmend, im Wege stehen kann, soll es nicht das vorrangige Ziel dieser Arbeit sein, die mannigfaltigen Spekulationen bezüglich der Gelungenheit des Doktor Faustus um eine weitere, gar als endgültig und abschließend sich gerierende Interpretation zu ergänzen. Dieser Anspruch wäre angesichts der Komplexität, Kompliziertheit und der prinzipiellen interpretatorischen Unerschöpflichkeit des zur Untersuchung stehenden Werkes überzogen und verfehlt. Nichtsdestoweniger ist es jedoch Anspruch und Ziel der vorliegenden Arbeit, vor allem zwei Gegenstandsbereiche, die in der umfangreichen und intensiven Faustus-Forschung bisher nicht in angemessener und überzeugender Weise Berücksichtigung gefunden haben, in den Blick zu nehmen. Ein erstes analytisches Desiderat umreißt Jürgen H. Petersen, wenn er folgende Aufgabe aus seinem Resümee der derzeitigen Forschungslage ableitet: "Vor allem müßte viel stärker, als es meines Wissens bisher geschehen ist, die Ambivalenz alles [im Roman; GK] Gesagten, die Ambivalenz der Darstellung selbst Berücksichtigung finden, das Augenmerk müßte sich mehr auf Zeitblom richten."

Was Petersen als die "Darstellung selbst" bezeichnet (in Abgrenzung vom Dargestellten) kann hier (in Abgrenzung von der Erzählung und von der Geschichte $^{9}$ ) als der Akt des Erzählens, als Narration verstanden werden, der bzw. die im Doktor Faustus zunächst natürlich untrennbar mit der erzählenden Instanz im Roman, d.h. mit Zeitblom, verknüpft ist. Lag in den älteren Arbeiten zum "Faustus" der Schwerpunkt des Interesses - diese ein wenig holzschnittartige Einteilung sei hier zunächst gestattet - vor allem auf der Figur des deutschen Tonkünstlers Leverkühn und somit vorrangig auf der Erzählung und der Geschichte des Romans, so ist Zeitblom zwar mittlerweile durchaus verstärkt in den Fokus der literaturwissenschaftlichen Aufmerksamkeit gerückt, jedoch verbleibt die Analyse dabei zumeist auf der Ebene der Erzäh- 
lung. D.h. der scheinbar so dilettantische Studienrat wird lediglich als eine auf der Ereignis-Ebene des Romans in das Erzählte involvierte Figur thematisiert (so geschehen etwa bereits ein Jahr vor dem Erscheinen von Petersens zitiertem Fazit in einer Untersuchung von Hans Hilgers ${ }^{10}$ ). Seine Rolle als Erzähler indes, mithin die strategische Art und Weise, in der Thomas Mann den Akt des Erzählens durch das narrative Medium Zeitblom gestaltet, und die aus dieser Gestaltung der Erzählsituation resultierenden, ambivalenten Konsequenzen bleiben bisher weitestgehend unterbelichtet.

Demzufolge besteht ein Hauptanliegen dieser Arbeit in der differenzierten Analyse und kritischen Diskussion der den Roman prägenden Gestaltung des Erzählaktes. Untersucht wird also jenes reichhaltige Repertoire von Erzählstrategien, das Mann im Doktor Faustus entfaltet, um die Realisierung der Geschichte, die dem Roman zugrundeliegt, zu gewährleisten. Unter der Realisierung der Geschichte soll hier die vom Autor grundsätzlich intendierte Parallelisierung der verschiedenen Stoff- und Strukturschichten ${ }^{11}$ des Romans verstanden werden, d.h. die analogisierende Verknüpfung der Strukturschicht des Künstlerromans mit den Schichten eines Faustromanes, eines Gesellschaftsromanes und eines Deutschlandromans. Eine Untersuchung der Erzählstrategien Manns würde indes in selbstzweckhafter, formalistischer Repetition erzähltechnischer Mittel befangen bleiben und somit einer lediglich narratologisch verbrämten Rückkehr zur werkimmanenten Interpretation Vorschub leisten, würde sie die Frage nach dem Wie nicht mit denen nach dem Warum und dem Was verbinden: Gefragt werden soll somit auch nach dem Bezug dieser Erzählstrategien zu den in der Grundkonzeption Manns entwickelten Stoff- und Strukturschichten des Romans, d.h. nach dem Verhältnis der Narration zu der der Erzählung zugrundeliegenden Geschichte. Im Vordergrund steht deshalb zunächst die Frage nach dem Ermöglichungszusammenhang eben dieser Geschichte (unter stetiger Berücksichtigung der konkreten Entfaltung derselben in der Erzählung). 
Die Frage nach dem Ermöglichungszusammenhang der Geschichte verweist indes auf ein zweites Desiderat innerhalb der Faustus-Forschung. Daß Literatur, mithin also auch die sogenannte "Höhenkamm"-Literatur weder nur aus Literatur, noch bloß aus der individuell-biographischen Disposition eines Autors oder den ihn beeinflussenden ideengeschichtlichen Impulsen entsteht, ist eine Einsicht, der sich die vorliegende Arbeit verpflichtet zeigt. Dies bedeutet natürlich keineswegs, daß die im Doktor Faustus virulenten innerliterarischen, biographischen und ideengeschichtlichen (Rück-)Bezüge hier ausgeblendet werden sollen. Die einsichtige These Bourdieus jedoch, derzufolge "sich in den Kunstwerken die sozialen Denkformen einer Epoche am elementarsten und vollständigsten aus[drücken]"12, ist hinsichtlich Manns Altersroman noch nicht hinreichend fruchtbar gemacht worden. Die mentalitätengeschichtliche ${ }^{13}$ Einbettung der Grundkonzeption des Romans, die Situierung der Bestandteile der Geschichte innerhalb des "kulturellen Kräftefelds"'14 der ersten Hälfte des 20. Jahrhunderts, ist meines Wissens bisher noch nicht grundlegend analysiert worden und bedarf deshalb einer detaillierteren Erläuterung. Denn, so konstatiert Bourdieu in diesem Zusammenhang treffend,

[...] noch seine [des Künstlers; GK] intellektuellen Entscheidungen unterliegen stets seiner Bildung und seinem Geschmack, den Internalisierungen der objektiven Kultur einer Gesellschaft, Epoche oder Klasse. Die Bildung (culture), wie sie sich in seinen Produktionen niederschlägt, ist kein Irgendetwas, das sich, wer weiß wie, hinter schon eine präexistente Intention hängte und damit deren Realisierung gegenüber äußerlich bliebe, sondern bildet im Gegenteil die transzendentale Bedingung der konkreten Konstitution einer künstlerischen Intention in einem Werk, genauso wie die Sprache als "gemeinsamer Sprachschatz" noch die Formulierung des ausgefallensten Wortes bedingt. ${ }^{15}$

Es scheint jedoch nicht überflüssig zu sein, noch einmal dezidiert darauf hinzuweisen, daß im Zusammenhang dieser Untersuchung angesichts der mentalitätengeschichtlichen Ausgangsfragestellung, die jene trans-individuellen Denkformen und Weltdeutungsmuster thematisiert, innerhalb derer die Grundkonzeption des Romans erst ermöglicht und verständlich wird, die eingehende und möglichst genaue Analyse der Erzählung und des Erzählaktes keineswegs 
obsolet wird. Die mentalitätengeschichtlich orientierte Rekonstruktion extratextueller Ermöglichungsfaktoren dispensiert die Literaturwissenschaft m.E. weder von einer intensiven Lektüre der Texte selbst, noch auch von jener hermeneutischen Anstrengung der Interpretation, die man als anachronistisch und unhaltbar zu perhorreszieren sich in den vergangenen Jahrzehnten allzu vorschnell und pauschalierend anbequemt hat. In der Gesamtanlage der vorliegenden Arbeit manifestiert sich also auch das methodologische Ansinnen, mentalitätengeschichtliche, ideengeschichtliche, (werk-)biographische und narratologische Herangehensweisen wechselseitig einander erhellend zu verbinden, in der Hoffnung, durch diesen (forschungs-)pragmatischen Eklektizismus methodisch bedingte Aspekt-Monismen weitestgehend zu vermeiden und $\mathrm{zu}$ einer möglichst perspektivenreichen und zugleich differenzierten Analyse des Untersuchungsobjektes zu gelangen.

Demzufolge sollen in Kapitel I, das die Ebene der Geschichte des Romans fokussiert, zunächst die Entwicklung der auch für die formale Gestaltung des Romans bedeutenden Grundkonzeption und die aus ihr resultierenden hybriden Strukturschichten thematisiert werden.

Kapitel II erörtert den mentalitätengeschichtlichen, und darin eingebettet den ideengeschichtlichen und (werk-)biographischen Ermöglichungszusammenhang des Romans bzw. seiner einzelnen Strukturschichten. Damit zusammenhängend wird die, aus der überdehnten Romankonzeption resultierende, Problematik der romaninternen Gestaltung der Parallelisierung der Strukturschichten auf der Ebene der Erzählung diskutiert. Diese Erörterung wird geleitet von der Hypothese, daß die hybride Romankonzeption im Roman selbst, vor allem durch die vom Autor angestrebte epische Exaktheit, zu einem strukturellen Defizit an Stimmigkeit führt. Dieses Defizit wiederum zeitigt eine immense Intensivierung und Komplizierung der von Mann angewendeten Erzählstrategien.

Nach einer thesenartigen Zusammenfassung der Ergebnisse wendet sich das III. Kapitel, in dem Handhabung, Funktion und Wirkung der Erzählstrate- 
gien und ihr Zusammenhang mit dem Mann'schen Romanverständnis analysiert werden, der Ebene des Erzählaktes zu. Zunächst soll anhand einiger von G. Genette und von J.H. Petersen entwickelten Kategorien des Erzählens eine grundlegende Analyse der Erzählsituation, die hauptsächlich durch die Einschaltung des narrativen Mediums Zeitblom gekennzeichnet ist, vorgenommen werden. Aufgezeigt werden sollen zugleich die Konsequenzen, die die strategische Gestaltung der Erzählsituation im Doktor Faustus für die Deutungsmöglichkeit des Romangeschehens mit sich bringt. Im Anschluß daran (III.4) folgt eine Analyse und Erörterung der - so die Arbeitshypothese dieses Kapitels für die formale Gestaltung des Romans maßgeblichen sogenannten LeitmotivTechnik Manns. Begriff, Ermöglichungszusammenhang, die intensive Art der Anwendung dieser Technik im Roman und ihre Konsequenzen sollen erörtert werden. Da zur Erläuterung der Leitmotivik eine in der bisherigen Forschungsliteratur nur in geringfügigem Maße erfolgte Exemplifizierung der leitmotivischen Vernetzungsstrategie am Text selbst angestrebt wird, erscheint häufigeres, belegendes Zitieren aus dem Roman notwendig. Kapitel III.5 untersucht und erläutert einige Phänomene der Transtextualität im Doktor Faustus, d.h. jene Erzählstrategien, die Mann selbst als eine Art des "höheren Abschreibens" ${ }^{16}$ bezeichnet: das mythologisierende Erzählen, die "Montage", intertextuelle Anspielungen und das Pastiche. Den Abschluß des dritten Kapitels bildet die schlaglichtartige, d.h. anhand einiger Beispiele ausgeführte Erörterung der Erzählstrategie der "doppelten Optik".

In Kapitel IV soll eine Strategie post festum Manns untersucht werden: die der Rezeptionslenkung durch nachträgliche Kommentierung des eigenen Werkes. Diese Strategie des Autors kann in unserem Zusammenhang, da sie über die drei Ebenen der Erzählung selbst hinausgreift, auch als MetaErzählstrategie, d.h. als ein Erzählen über das und nach dem Erzählen charakterisiert werden. Sie soll am Beispiel einiger, vor allem auch in der Entstehung des Doktor Faustus (1949) plazierter Selbstaussagen bezüglich des Doktor Faustus verdeutlicht werden. Diese Kommentare, und auch dies wird von der 
bisherigen Faustus-Forschung zumeist übersehen, zeugen vom Bestreben Manns, seinen Altersroman durch vergleichende Bezugnahme auf das Werk von James Joyce, vor allem jedoch auf dessen 25 Jahre vor dem Faustus erschienenen Ulysses, in den Diskurs über die literarische Moderne einzuordnen.

Selbstaussagen Manns in Briefen oder Kommentaren werden im Rahmen dieser Arbeit stets auch als semi-literarische rezeptionslenkende Erzählstrategien interpretiert, d.h. sie sind als Beleg oder Untermauerung für eigene Thesen prinzipiell mit Vorsicht zu genießen. Verwendbar zumindest zur Bekräftigung eigener Aussagen erscheinen im Rahmen dieser Arbeit indes Tagebuchaufzeichnungen Manns, die in der Zeit während der Arbeit am Roman entstehen, da diese, auch wenn der Autor sich ihrer späteren Veröffentlichung durchaus bewußt sein mag, weniger den Charakter einer bewußt inszenierten Rezeptionslenkung, als vielmehr den Status einer mitunter von extremen Zweifeln zeugenden (Selbst-)Vergewisserung des Autors über sein entstehendes Werk haben. ${ }^{17}$

Kapitel IV fährt fort mit einem skizzenhaften Exkurs zum Vergleich zwischen Joyces Ulysses und Manns Doktor Faustus, der die in der zusammenfassenden Schlußbetrachtung geführte Diskussion um die Möglichkeiten einer gattungsgeschichtlichen Einordnung des Mann'schen Romans zwischen Tradition und Moderne vorbereitet.

Als Schlußbetrachtung und Zusammenfassung fungiert diese Diskussion insofern, als die Problematik der vom Autor intendierten und durch intensiven Einsatz der erörterten Erzählstrategien forcierten Parallelisierung noch einmal aufgegriffen wird. Abschließend soll gezeigt werden, welche möglichen Konsequenzen die in Kapitel II entfaltete Hypothese von einem strukturellen Stimmigkeitsdefizit des Romans für die gattungsgeschichtliche Stellung des Doktor Faustus hat:

Möglicherweise erscheint der Roman gerade in seinem erzählstrategisch virtuosen Scheitern daran, Disparates ästhetisch zu harmonisieren, d.h., die Desintegrationen einer modernen Welt zumindest in der bis zum Äußersten 
gespannten, geschlossenen Form des Kunstwerks noch aufzuheben, als literarisches Dokument einer ungewollten Modernität.

Abschließend noch drei Hinweise zur Textgestaltung: Natürlich ist sich der Verfasser der vorliegenden Arbeit der Tatsache bewußt, daß, wer heutzutage eine wie auch immer konzipierte wissenschaftliche Analyse des Doktor Faustus anstrebt, sich bis zu einem gewissen Grade des "In-Spuren-Gehens" nicht enthalten kann. Unweigerlich nämlich bewegt man sich in den Spuren jener für den Einzelnen mittlerweile kaum noch in ihrer Gesamtheit überschaubaren Forschungsliteratur zum Altersroman Thomas Manns. Dennoch oder gerade aufgrund der zunehmend unübersichtlichen und dispersen Forschungslage erscheint es ratsam, auf ein Kapitel, daß sich ausschließlich mit dem derzeit erreichten Forschungsstand auseinandersetzt, zu verzichten. Ein solches Unterfangen müßte zweifelsohne zu einem eigenständigen, anderen Buch anschwellen. Dieser Verzicht sollte jedoch nicht als sophistische Legitimation etwaiger Bequemlichkeiten mißgedeutet werden. Denn selbstverständlich finden auch in dieser Arbeit Bezugnahmen und kritische Auseinandersetzungen mit der zugänglichen Sekundärliteratur allenthalben statt. Prinzipiell drei Möglichkeiten der textuellen Integration stehen einem wissenschaftlichen Autor hinsichtlich des Umgangs mit seinen Vorgängern zur Verfügung:

1. Er integriert Bezugnahme und Kritik in den Haupttext, mit dem Resultat, daß dieser auf Kosten der Stringenz und der Lesbarkeit unnötig anschwillt und "ausfranst".

2. Er greift auf Fußnoten zurück, was zu der unästhetischen und darüberhinaus lächerlichen Konsequenz führt, daß der Fußnotentext den Haupttext verdrängt und überlagert.

3. Er fügt einen Anmerkungsteil am Ende des Textes an und muß sich der möglichen "Gefahr" bewußt sein, daß das ständige Hin-und-Her-BlätternMüssen den geneigten Leser seines Interesses an den Anmerkungen verlustig gehen läßt. 
Die vorliegende Arbeit versucht sich an einer vierten, vermutlich nicht minder beanstandenswerten Variante: Um die visuelle und inhaltliche Kohärenz der Untersuchung nicht unnötig zu beeinträchtigen, wird auf Fußnoten gänzlich verzichtet. Bezugnahmen auf die Sekundärliteratur, die affirmierenden oder affirmativen Charakters sind, finden sich weitestgehend in einen Anmerkungs(Endnoten-)Apparat eingearbeitet. Die mitunter dennoch notwendige kritische Diskussion einiger wichtiger Forschungspositionen wird zumindest dann, wenn die in dieser Arbeit postulierten Hypothesen und Einsichten gerade auf der Kontrastfolie der kritisierten Positionen, und somit gleichsam durch und gegen diese, klarere und schärfere Umrisse gewinnen, möglichst unaufdringlich in den Haupttext zu integrieren versucht.

Zitiert wird im Folgenden aus der Taschenbuchausgabe der Gesammelten Werke Thomas Manns (Fischer: Frankfurt am Main 1990), mit Rücksicht darauf, daß diese Ausgabe wahrscheinlich die meistverbreitete und zugänglichste ist. Die Fundstellen der Zitate aus dem Doktor Faustus (= Gesammelte Werke, Band VI = GW,VI) werden durch Angaben in Klammern jeweils nach dem Zitierten kenntlich gemacht. Die Belege anderer Zitate aus dem Werk Manns sind, wenn nicht ausdrücklich anders gekennzeichnet, jeweils im Anmerkungsapparat zu finden.

Die Entschlüsselung einiger weniger abgekürzt zitierter Titel ist anhand eines vor dem Literaturverzeichnis zu findenden Siglenverzeichnisses möglich. 